\title{
Can multi-proxy arrays improve environmental reconstructions and reveal past microbial interactions?
}

\section{EINAT SEGEV}

Weizmann Institute of Science

Presenting Author: einat.segev@weizmann.ac.il

Coccolithophores are a diverse group of calcifying microalgae that have left a prominent fossil record on Earth. Various coccolithophore relics, both organic and inorganic, serve as proxies for reconstruction of past oceanic conditions. Commonly used coccolithophore-derived proxies for past sea surface temperature are alkenone unsaturation $\left(\mathrm{U}^{\mathrm{K}}{ }_{37}\right)$, and the $\mathrm{Sr} / \mathrm{Ca}$ ratio of the coccolith calcite.

Emiliania huxleyi is the most widely distributed representative of the coccolithophores in modern oceans, and it is known to engage in dynamic interactions with bacteria. Algal-bacterial interactions influence various aspects of algal physiology and biochemistry.

I will present an experimental model system for the cocultivation of algae and bacteria that allows detailed characterization of microbial interactions. According to data collected during multiple experiments in this model system, algal-bacterial interactions influence alkenone unsaturation, thereby influencing temperature reconstruction. In contrast, the $\mathrm{Sr} / \mathrm{Ca}$ ratio of the coccolith calcite remains unaltered by bacteria, thus serving as a robust environmental paleo-proxy independent of microbial interactions.

I will discuss how the study of paleo-proxies in the context of microbial interactions has the potential to generate improved reconstructions of past environmental conditions. Moreover, multi-proxy combinations of both organic and inorganic indicators, emerge as a novel approach for detecting past microbial interactions. 